\title{
Uma proposta metodológica para avaliação de sistemas socioecológicos de manejo de pesca de pequena escala na Amazônia
}

\section{A methodological proposal for the evaluation of socioecological aspects of small scale fisheries management in the Amazon}

\author{
Urbano Lopes da Silva-Júnior ${ }^{1}$ \\ Antonio Francisco Perrone Oviedo ${ }^{2}$
}

\begin{abstract}
Resumo: Este artigo propõe uma abordagem comparativa para a avaliação de sistemas de manejo de pescarias de pequena escala na Amazônia brasileira. Esta abordagem se baseia na estrutura do sistema socioecológico (SES), adotada para explicar as condições necessárias para a sustentabilidade e a cooperação dos usuários na gestão de recursos naturais, como uma alternativa mais adequada às abordagens baseadas apenas em direitos de propriedade ou intervenção governamental. No entanto, os gestores e usuários geralmente não possuem as informações necessárias compiladas e disponíveis para um SES específico, enquanto algumas ações precisam ser tomadas imediatamente. Assim, uma proposta metodológica para avaliação expressa das variáveis do SES pode ser útil para avaliar o desempenho de um sistema socioecológico e indicar ações para promover sua sustentabilidade, afastando-o assim da metáfora da "tragédia dos comuns". Referências de sustentabilidade socioecológica, insustentabilidade ecológica e insustentabilidade sociocultural baseado nesta metodologia são sugeridas. A adoção de uma estrutura de SES baseada em variáveis chaves pode ser útil para a gestão de sistemas de pesca de pequena escala até que estudos interdisciplinares completos sejam disponibilizados.
\end{abstract}

Palavras-chave: sistema socioecológico, avaliação de sustentabilidade, pesca de subsistência, Amazônia.

\begin{abstract}
This article proposes a comparative approach for evaluating smallscale fisheries in the Brazilian Amazon. This approach is based on the general framework of socioecological systems (SES), adopted to explain the conditions necessary for sustainability and cooperation of users in the management of natural resources, as a better alternative to approaches based on property rights or governmental intervention. However, managers and users often do not have the necessary information compiled and available for a specific SES, while some actions need to be taken immediately. Thus, a methodological proposal for the
\end{abstract}

\footnotetext{
${ }^{1}$ Possui graduação em Ecologia pela Universidade Estadual Paulista Júlio de Mesquita Filho - UNESP, mestrado e doutorado em Biologia de Água Doce e Pesca Interior pelo Instituto Nacional de Pesquisas da Amazônia - INPA (BADPI/INPA). E-mail: urbanosljr@gmail.com

${ }^{2}$ Pós-doutorado em políticas públicas e gestão ambiental pelo Centro de Desenvolvimento Sustentável (CDS) da Universidade de Brasília UnB). Doutor pelo CDS/UnB e Mestre em Geografia pela Universidade de São Paulo. Graduado em Ciências Agrárias pela Universidade de Taubaté. E-mail: antoniopoviedo@gmail.com
} 
express evaluation of SES variables can be useful to evaluate performances of a socioecological system and indicate actions to promote its sustainability, thus removing it from the metaphor of the "tragedy of the commons". References to social-ecological sustainability, ecological unsustainability and social-cultural unsustainability based on this methodology are suggested. The adoption of SES framework based on key variables may be useful for small-scale management of fisheries until interdisciplinary studies are completed.

Keywords: social-ecological system, sustainability assessment, subsistence fishing, Amazon.

\section{Introdução}

Um dos principais desafios científicos para o uso sustentável dos recursos comuns tem sido a integração adequada de disciplinas que lidam com as dimensões biofísica e humana (STERNLIEB et al., 2013; KIRK-LAWLOR \& ALLRED, 2017; BOILLAT et al., 2017). A abordagem econômica tradicional para a gestão de recursos comuns propõe que se todos os atores se comportarem de acordo com seus próprios interesses, isto levaria ao seu uso excessivo e consequente colapso (HARDIN, 1968). Ainda, existe uma tendência conceitual de abordar as interações homem-ambiente de forma simplificada, sugerindo "soluções universais" para o manejo dos recursos naturais, onde os contextos ecológicos e sociais apresentam características muito distintas (PRITCHETT \& WOOLCOCK, 2004; WILSON et al., 2013).

No entanto, Ostrom (1990) demostrou que os indivíduos são capazes de se autoorganizar e governar com sucesso os recursos comuns. Tal abordagem promoveu o desenvolvimento conceitual dos sistemas socioecológicos (SES) (OSTROM, 2009) com implicações para as políticas públicas de gestão de recursos naturais, ajudando a explicar a ineficácia de muitos regimes de governança (ANDERIES \& JANSSEN, 2013).

As pesquisas de campo com o SES indicaram que existem vários fatores que explicam o sucesso na gestão de recursos comuns (COX et al., 2010; FREY \& RUSCH, 2013). Ostrom (1999) definiu um conjunto de fatores de sucesso que ela chamou de princípios de design: "um elemento ou condição essencial que ajuda a explicar o sucesso dessas instituições na manutenção de recursos comuns". Posteriormente, tais fatores foram incorporados no concito dos SES.

Vários estudos têm usado ou discutido esses fatores de sucesso e suas con- 
tribuições para a cooperação entre usuários de recursos comuns (AGRAWAL, 2001; AGRAWAL \& CHHATRE, 2006; COX et al., 2010). Um estudo utilizou 25 iniciativas de pesca e avaliou os princípios definidos por Ostrom (1999), onde os limites claros sobre o espaço geográfico dos recursos comuns e o número de usuários representaram os princípios mais relevantes (POMEROY et al., 2001). Adicionando a dimensão da política, GARRETTA et al. (2012) examinaram o papel dos espaços de participação no incentivo à compreensão compartilhada entre os usuários. A percepção dos usuários sobre as políticas de uso de recursos naturais oferece aos gestores subsídios para a formulação de políticas e maior eficiência do sistema de manejo (DELANEY \& HADJIMICHAEL, 2017). Para melhor desempenho das políticas públicas, os usuários e gestores precisam estar envolvidos no processo de tomada de decisão (MARSHALL, 2007; BRONDíZIO et al., 2009; NUNAN et al., 2012).

Uma etapa em direção à modelagem do SES é realizada por meio da organização das variáveis em uma estrutura multinível. O uso dessa estrutura permite que os usuários e gestores criem um quadro de análise sobre como as variáveis do sistema ecológico, da unidade de recursos, dos usuários e do sistema de governança interagem uns com os outros e os resultados dessa interação (OSTROM, 2007). Além disso, os usuários e gestores podem usar essa estrutura para avaliar o efeito e a interação dessas variáveis nos cenários econômico, político e ecológico (OSTROM et al., 2007). Tal estrutura permitir uma integração entre conhecimento local e a ciência acadêmica. Essa integração permite a construção de diagnósticos que combinam os arranjos de governança com problemas específicos em um contexto socioecológico (OSTROM, 2007).

Um instrumento de avaliação de sustentabilidade tendo como base o conceito de SES necessita de dados quantitativos e qualitativos. Estudos multidisciplinares devem ser conduzidos para compor o conjunto de dados necessários e adequados para avaliar a interação entre os gestores e os usuários locais (OSTROM et al., 2007; KHOLER \& BRONDÍZIO, 2017). Dados qualitativos mostram que as atitudes e o comportamento dos atores sociais envolvidos são possíveis indicadores para avaliar o desempenho das políticas públicas de gestão ambiental (ASAH, 2008; PETURSDOTTIR et al. 2013). O estudo de Petursdottir et al. (2013) também sugere que as limitações na governança podem afetar os resultados das políticas relacionadas ao gerenciamento de recursos comuns. 
Gutiérrez et al. (2011) examinaram 130 pescarias co-manejadas em diversos países com diferentes condições sociais, econômicas e ecológicas. O estudo considerou variáveis de co-gestão relacionadas às variáveis sugeridas por Ostrom (2009). A liderança foi identificada como uma variável chave que contribui para a co-gestão, bem como as quotas de pesca, coesão social e direito de propriedade. Os autores concluíram que os mecanismos de implementação, as políticas de gestão de longo prazo e as informações sobre recursos representaram variáveis menos importantes.

A abordagem SES para a gestão da pesca apresenta uma enorme perspectiva para alcançar a sustentabilidade (GUTIÉRREZ et al., 2011; KITTINGER et al., 2013). No entanto, a natureza complexa do contexto socioambiental onde as variáveis do SES interagem limita a identificação das condições e tendências do SES para os gerentes e usuários (OLSSON et al., 2004; ASAH, 2008). Com isso, existe uma necessidade por indicadores simplificados e facilmente interpretáveis (CARPENTER et al., 2001), bem como que representem contextos locais (BEROYA-EITNER, 2016). A geração de conhecimento sobre SES integrada com práticas de gestão é uma abordagem cada vez mais proposta, evoluindo com o quadro institucional e o processo de gestão adaptativa (WALKER et al., 2002; OLSSON et al. 2004).

A avaliação da sustentabilidade usando variáveis do SES tem sido utilizada como uma ferramenta para o desenvolvimento políticas públicas voltadas para a melhoria ambiental, econômica, social ou tecnológica (SINGH et al. 2012; HINKEL et al., 2015). Desde sua primeira publicação (OSTROM, 2007), o quadro analítico para o SES tem sido atualizado (OSTROM 2009, 2011; MCGINNIS e OSTROM 2014) e refinado por meio do fornecimento de critérios claros para ordenar variáveis em camadas, inclusão de novos conceitos e definição de métricas de avaliação (HINKEL et al., 2014; DURAIAPPAH et al., 2014). Para a gestão da pesca, tem sido importante na análise da sustentabilidade e cooperação entre pescadores, usando diferentes abordagens metodológicas que vão desde estudos de caso (BASURTO e OSTROM 2009; ASWANI et al. 2013; ERNST et al. 2013) a estudos de larga escala (GUTIÉRREZ et al. 2011; CINNER et al. 2012; MACNEIL e CINNER 2013). Para ir além das "soluções universais" e construir métodos diagnósticos mais efetivos, SES propõe a identificação de combinações de variáveis que afetam os recursos comuns. 
As variáveis do SES, como indicadores de desempenho do sistema, podem ajudar a criar consenso entre os gestores e usuários, facilitando o compartilhamento do conhecimento (BAULER, 2012). Este processo de análise das variáveis específicas do SES pode, assim, ganhar importância como ferramentas de gestão ao reconhecer sua inserção nas instituições envolvidas. Esta abordagem pode adaptar continuamente o processo institucional de avaliação de sustentabilidade (CONNOR e DOVERS, 2004; OSTROM, 2007), uma vez que mantém as variáveis incorporadas nos espaços de tomada de decisão.

Existe um amplo reconhecimento do valioso papel que o conhecimento ecológico local pode desempenhar na gestão dos recursos comuns (BUTLER et al., 2012). Este conhecimento consiste nas habilidades práticas adquiridas por meio de atividades de subsistência e no conhecimento coletivo adquirido pela comunidade através de gerações (BERKES et al., 2000; INGOLD, 2011). O conhecimento local das espécies, suas histórias de vida, distribuições, características ambientais são aninhados dentro de sistemas, ferramentas e medidas de gerenciamento de recursos. Por sua vez, este conhecimento está incorporado nas instituições, códigos, normas e procedimentos de tomada de decisão necessários para implementar sistemas de gestão (OTEROS-ROZAS et al., 2013). Em muitos casos, a combinação entre o conhecimento ecológico local e a ciência acadêmica levou à criação de sistemas de gestão eficientes (FOLKE et al., 2005; BERKES, 2009; RAYMOND et al., 2010; TERER et al., 2012). Johannes (1998, 2002) e Johannes et al. (2000) sugerem que o esgotamento dos estoques pesqueiros, informações limitadas e o baixo grau de governança são fatores que alavancam a integração do conhecimento. De acordo com Garibaldi e Turner (2004) e Castello et al. (2011), lidar com "espécies-chave culturais" pode facilitar esse tipo de integração.

Stephenson et al.(2018) através de um processo participativo com diversos atores e através do conceito de SES, prorpuseram um arcabouço do mesmo específico para a gestão pesqueira, visando corrigir o desbalanceamento do peso das dimensões humanas da sustentabilidade quando comparada o da dimensão biológica. Tal arcabouço é proposto tendo em vista a definição de obejtivos na implementação da gestão pesqueira, tendo, portanto, caráter prognóstico sendo sua aplicação limitada pelas condição políticas e institucionais do país em questão (STEPHENSON et al, 2018). 
Em outro sentido, mas de forma complementar, a proposta do presente trabalho baseada na abordagem do SES é de caráter diagnóstico em situaçções práticas no contexto da pesca de pequena escala na região amazônica. Desta forma o presente artigo tem como objetivo propor uma abordagem para designar valores para as variáveis-chave, tendo em vista a análise empírica da evolução do sistema de manejo da pesca.

\section{Metodologia}

Esta pesquisa avaliou até que ponto a abordagem das variáveis do SES pelos pescadores pode melhorar o sistema de manejo dos recursos pesqueiros. O quadro analítico do SES (OSTROM, 2009) foi utilizado para reunir o conhecimento multidisciplinar da pesca, a fim de facilitar a avaliação e identificar combinações de variáveis que afetam as interações e os resultados do sistema específico de manejo. Esta decomposição do sistema pesqueiro é essencial para alcançar uma melhor compreensão do SES (OSTROM, 2007) e criar alternativas para a melhoria de sua performance. A primeira etapa é a decomposição conceitual das variáveis em classes e subclasses. A segunda é a identificação de subsistemas relativamente independentes, mas que afetam o desempenho uns dos outros. Este quadro analítico (Figura 2) baseia-se na relação entre quatro subsistemas do SES:

(i) Sistema do Recurso (RS): um determinado território contendo o recurso comum;

(ii) Unidade do Recurso (RU): unidade de recurso comum mais utilizado;

(iii) Usuários (U): conjunto de indivíduos que usam o território (RS) para diferentes propósitos;

(iv) Sistema de governança (GS): agências governamentais e outras organizações envolvidas na gestão do sistema (RS), suas normas e regras específicas, e como elas são construídas.

Os subsistemas do SES são conectados por um conjunto de interações (I) produzindo um conjunto de resultados (O) que, por sua vez, fornecem feedback aos subsistemas, ecossistemas associados (ECO) e configurações sociais, econômicas e políticas (S).

O quadro analítico do SES visa fornecer algum suporte para a avaliação da sustentabilidade dos mecanismos de gestão da pesca, abordando as seguintes questões 


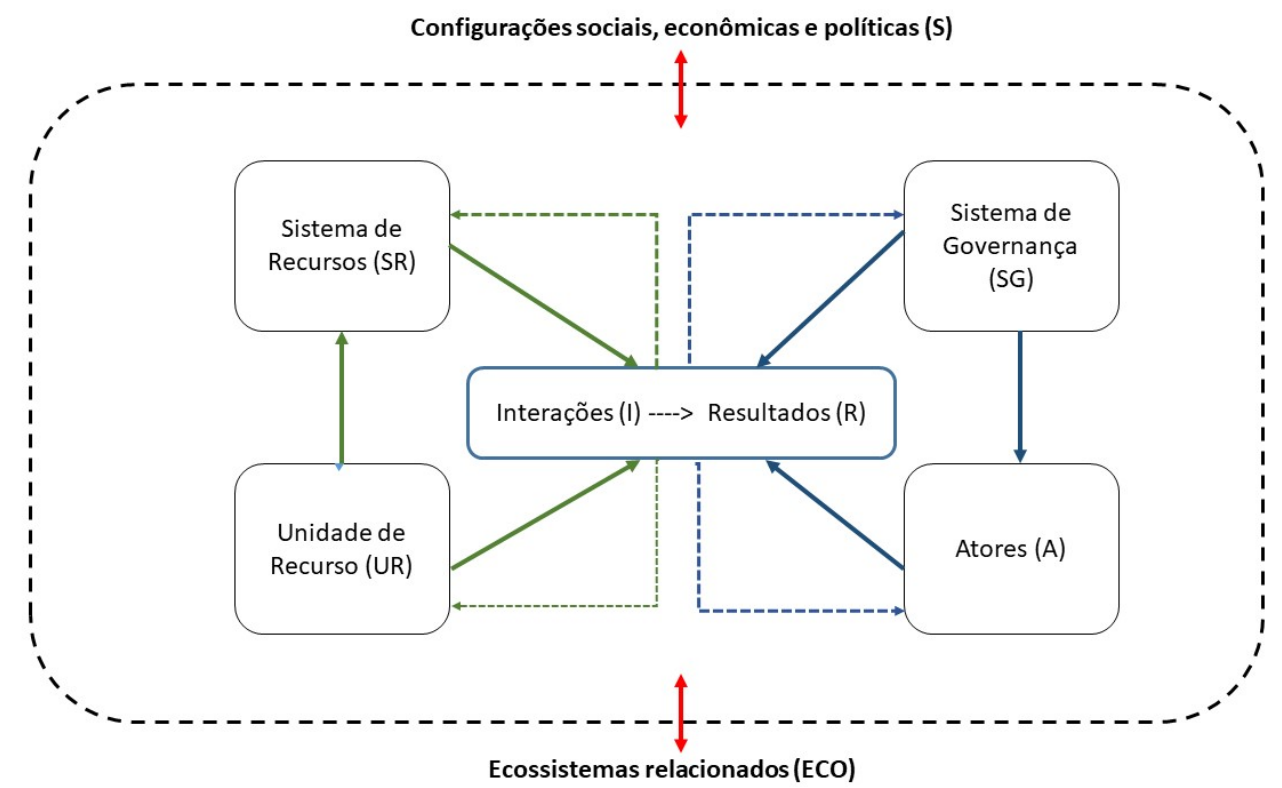

Figura 1. Quadro analítico do sistema socioecológico. Adaptado de Ostrom (2009).

relacionadas aos subsistemas do SES:

1. (RS) - Quais são as principais propriedades e a dinâmica do ecossistema em questão? Que papel desempenha a pesca em seus fluxos de energia? (ECO) Como o ecossistema é afetado por eventuais externalidades?

2. (RU) - Como os diferentes cenários de manejo da pesca podem afetar a população de peixes e as propriedades do ecossistema? (ECO) - Que tipo de externalidades cada cenário de manejo pode gerar?

3. (RS) e (RU) como condições de contorno são favoráveis, em termo de produção e previsibilidade, para contribuir para governança (GS) e desenvolvimento local sustentável (U)?

Cada um dos subsistemas mencionados acima é composto por variáveis de segundo nível, conforme descrito por Ostrom et al. (2007) e Ostrom (2009). Algumas variáveis foram consideradas neste estudo como variáveis-chave relacionadas à criação de condições de cooperação entre os usuários (Tabela 1).

A obtenção de informações para avaliação de cada uma das variáveis do SES é o primeiro passo para a compreensão das condições necessárias para os usuários de um determinado sistema de manejo da pesca se auto-organizarem. Tal análise deve 
Tabela 1. Conjunto de variáveis-chave e respectivos efeitos, conforme indicado por Ostrom et al. (2007) e Ostrom (2009).

\begin{tabular}{|c|c|c|c|}
\hline Subsistema & $\begin{array}{l}\text { Variáveis- } \\
\text { chave }\end{array}$ & Efeito & $\begin{array}{l}\text { Aproximação matemática } \\
\text { do efeito } \mathbf{f} P_{\text {Cooperao }}= \\
f(\text { varivel }- \text { chave })\end{array}$ \\
\hline RS3 & $\begin{array}{ll}\text { Tamanho } & \text { do } \\
\text { sistema } & \text { de } \\
\text { recursos } & \end{array}$ & $\begin{array}{l}\text { O tamanho territorial moderado é mais } \\
\text { propício a cooperação. }\end{array}$ & Parábola negativa \\
\hline RS5 & $\begin{array}{l}\text { Produtividade } \\
\text { do sistema }\end{array}$ & $\begin{array}{l}\text { Um recurso em situação extrema, esgo- } \\
\text { tado ou muito abundante, não será atra- } \\
\text { tivo para a gestão. Produção moderada } \\
\text { demonstrando possível escassez, irá pro- } \\
\text { mover maior interesse entre os usuários. }\end{array}$ & Parábola negativa \\
\hline RS7 & $\begin{array}{l}\text { Previsibilidade } \\
\text { da dinâmica do } \\
\text { sistema }\end{array}$ & $\begin{array}{l}\text { O sistema deve ser previsível o suficiente } \\
\text { para que os usuários possam estimar os } \\
\text { efeitos de suas regras. }\end{array}$ & Linear positiva \\
\hline RU1 & $\begin{array}{ll}\text { Mobilidade } & \text { da } \\
\text { unidade } & \text { de } \\
\text { recurso } & \end{array}$ & $\begin{array}{l}\text { Recursos com alta mobilidade exigem al- } \\
\text { tos custos de monitoramento. }\end{array}$ & Linear negativa \\
\hline GS6 & $\begin{array}{l}\text { Regras de es- } \\
\text { colha coletiva }\end{array}$ & $\begin{array}{l}\text { Se os usuários têm a legitimidade e au- } \\
\text { tonomia para criar e para impor as suas } \\
\text { regras, eles estarão mais motivados para } \\
\text { a auto-organização e proteção dos recur- } \\
\text { sos contra usuários externos. }\end{array}$ & Linear positiva \\
\hline U1 & $\begin{array}{l}\text { Número de } \\
\text { usuários }\end{array}$ & $\begin{array}{l}\text { Depende de sua relação com RS3. Ge- } \\
\text { renciar muitos usuários em um território } \\
\text { relativamente pequeno pode não ser ren- } \\
\text { tável. Por outro lado, em território grande } \\
\text { um grande número de usuários pode ser } \\
\text { útil para as atividades de monitoramento, } \\
\text { por exemplo. }\end{array}$ & $\begin{array}{l}\text { "Parábola negativa em rela- } \\
\text { ção à (U1/RS3)"razão }\end{array}$ \\
\hline U5 & Liderança & $\begin{array}{l}\text { Quando alguns usuários são respeitados } \\
\text { como líderes e têm competências empre- } \\
\text { sariais, a cooperação entre os usuários é } \\
\text { mais provável. }\end{array}$ & Linear positiva \\
\hline U6 & $\begin{array}{l}\text { Normas/capital } \\
\text { social }\end{array}$ & $\begin{array}{l}\text { Existência prévia de padrões éti- } \\
\text { cos/morais que determinam as normas } \\
\text { de reciprocidade entre os usuários irá } \\
\text { diminuir os custos de transação em } \\
\text { iniciativas de longo prazo. }\end{array}$ & Linear positiva \\
\hline U7 & $\begin{array}{l}\text { Conhecimento } \\
\text { do SES }\end{array}$ & $\begin{array}{l}\text { Quando os usuários compartilham conhe- } \\
\text { cimento comum de atributos relevantes do } \\
\text { SES, eles percebem uma redução nos } \\
\text { custos de organização e operacionaliza- } \\
\text { ção das ações. }\end{array}$ & Linear positiva \\
\hline U8 & $\begin{array}{l}\text { Importância do } \\
\text { recurso para o } \\
\text { usuário }\end{array}$ & $\begin{array}{l}\text { Os custos na auto-organização e coope- } \\
\text { ração são assumidos somente se: (i) o re- } \\
\text { curso desempenha um papel importante } \\
\text { para a subsistência ou (ii) usuários têm } \\
\text { algum valor social ou cultural reivindicado } \\
\text { para a sua sustentabilidade. }\end{array}$ & Linear positiva \\
\hline
\end{tabular}


reconhecer as interações entre variáveis. Além disso, essas interações ocorrem principalmente em um sistema não linear (JANSSEN, 2002; LEVIN, 1992). Anderies e Janssen (2013) desenvolveram uma abordagem didática para avaliar a estrutura do SES. No entanto, este artigo sugere um método sintético e de aplicação mais rápida para avaliar um determinado SES ao longo de um espectro multidimensional de sustentabilidade, baseado em uma abordagem empírica.

O modelo proposto adota como principal premissa que o usuário toma sua decisão de participar de um esquema de cooperação baseando-se na sua percepção de recompensa ao fazê-lo, pois na maioria das vezes ele não tem como fazer isto de uma forma objetiva. Colocando isto na linguagem da teoria dos jogos significa que a probabilidade de um determinado jogador (usuário) cooperar com os outros depende de como, a partir de percepção, ele deprecia suas recompensas do passado $(1-\alpha)$ e a recompensa potencial no futuro $(1-\beta)$ dentro de um determinado jogo de cooperação. Assim, a probabilidade de um usuário cooperar é baseada na sua percepção de uma recompensa geral $(\delta)$ que pode ser dada por:

$$
S=\frac{(1-\alpha)}{(1-\beta)}
$$

Onde $\alpha$ e $(1-\beta)$ são dependentes de diferentes formas de variáveis-chave propostas por Ostrom (2009):

$$
\begin{gathered}
\alpha=f(U 1, U 5, U 6, U 7, G S 6) \\
\beta=f(U 1, R S 3, R S 5, R S 7, R U 1, G S 6)
\end{gathered}
$$

Para demonstrar a aplicação desta metodologia foram utilizados três cenários hipotéticos que ilustram situações de manejo de pesca de pequena escala na Amazônia: 1) Sustentabilidade socioecológica 2) Insustentabilidade social e cultural, e 3) Insustentabilidade socioecológica.

Os valores de cada uma das variáveis-chave foram ponderados tendo o valor 1 
como ponto de referência, ou ponto de melhor performance, conforme aproximação matemática contida na Tabela 1 (Figura 2).

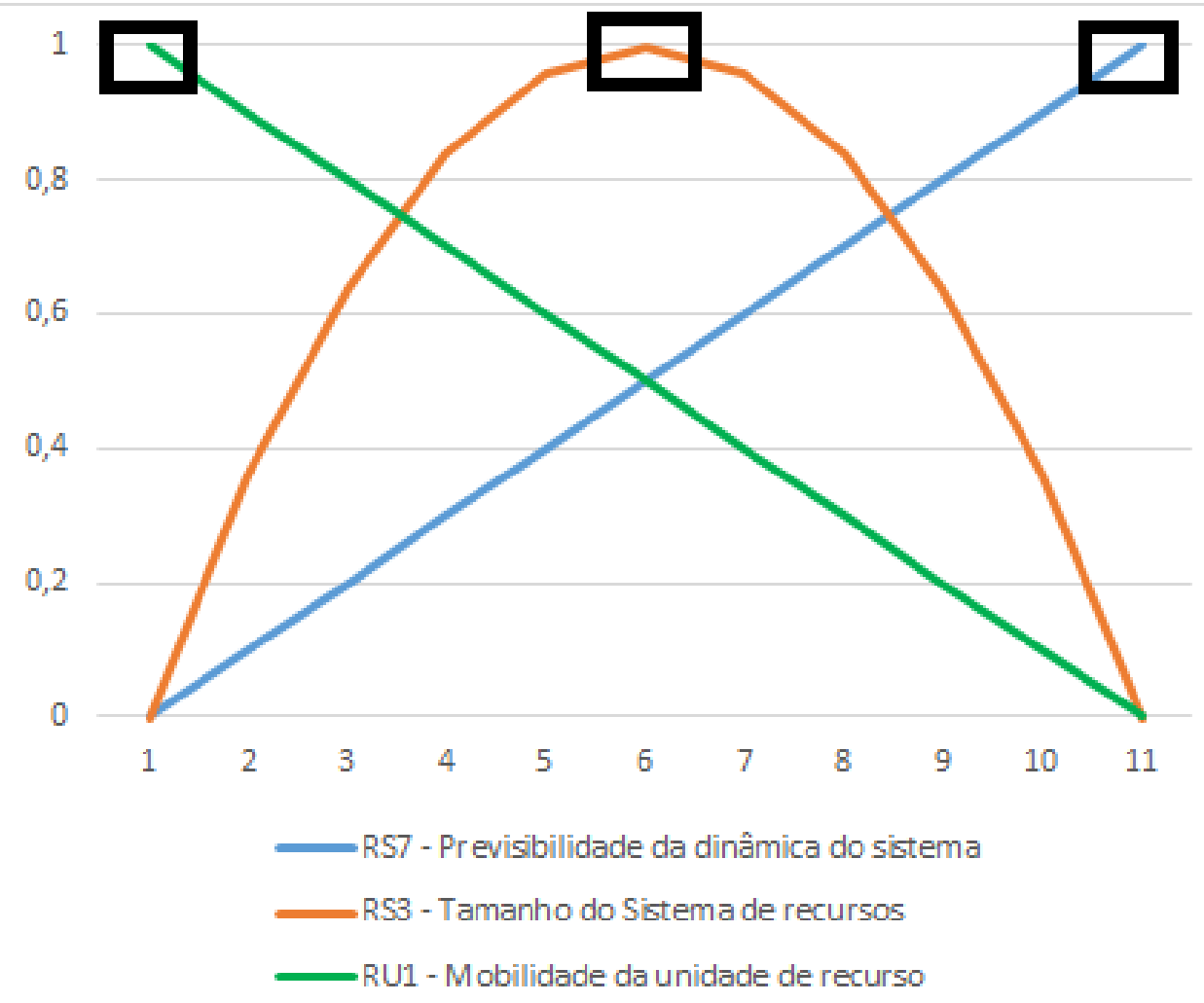

Figura 2. llustração demonstrando como cada uma das aproximações matemáticas da Tabela 1 podem funcionar para a atribuição de notas das variáveis-chave. Os retângulos negros representam o ponto de melhor performance (nota máxima) para três exemplos de varáveis-chave.

\section{Resultados}

Os resultados apresentados na Tabela 2 e na Figura 2 demonstram como cada um dos três cenários se apresentaria no contexto de avaliação das variáveis do SES, sugerindo referências comparativas a serem utilizadas em casos práticos.

Os valores obtidos para cada uma das variáveis, para uma avaliação rápida, podem ser obtidos pelo conhecimento empírico que se possui para cada um dos sistemas, ou através da aplicação de entrevistas estruturadas ou semi-estruturadas que aportem insumos para avaliação das variáveis-chave dos diferentes subsistemas. 
Tabela 2. Notas atribuídas ao conjunto de variáveis-chave para cada um dos cenários propostos.

\begin{tabular}{l|l|l|l|l}
\hline Subsistema & Variáveis-chave & $\begin{array}{l}\text { NOTA - Ce- } \\
\text { nário 1 }\end{array}$ & $\begin{array}{l}\text { NOTA - Ce- } \\
\text { nário 2 }\end{array}$ & $\begin{array}{l}\text { NOTA - Ce- } \\
\text { nário 3 }\end{array}$ \\
\hline RS3 & $\begin{array}{l}\text { Tamanho do sistema de re- } \\
\text { cursos }\end{array}$ & 0,9 & 0,8 & 1 \\
\hline RS5 & Produtividade do sistema & 0,9 & 0,5 & 0,7 \\
\hline RS7 & $\begin{array}{l}\text { Previsibilidade da dinâmica } \\
\text { do sistema }\end{array}$ & 0,9 & 0,9 & 0,9 \\
\hline RU1 & $\begin{array}{l}\text { Mobilidade da unidade de re- } \\
\text { curso }\end{array}$ & 0,9 & 0,9 & 0,9 \\
\hline U1 & Número de usuários & 0,9 & 1 & 0,8 \\
\hline U5 & Liderança & 0,9 & 0,7 & 0,5 \\
\hline U6 & Normas/capital social & 0,9 & 0,7 & 0,3 \\
\hline U7 & Conhecimento do SES & 0,9 & 0,5 & 0 \\
\hline \multirow{2}{*}{ I8 } & $\begin{array}{l}\text { Importância do recurso para } \\
\text { o usuário }\end{array}$ & 0,9 & 0,8 & 0,5 \\
\hline GS6 & Regras de escolha coletiva & 0,9 & 0,7 & 0,2 \\
\hline Alfa & & 0,8 & 0,1 & 0,1 \\
\cline { 3 - 5 } Beta & & 0,6 & 0,3 & 0,2 \\
\cline { 3 - 5 } Sigma & & 0,3 & 0,7 & 0,1 \\
\hline
\end{tabular}

\section{Discussão dos resultados}

Os cenários de sustentabilidade foram estimados com base em um conjunto particular de variáveis (Tabela 2). A geração do cenário de insustentabilidade socioecológica requer as seguintes suposições: (i) o sistema de recursos envolve extensas áreas de manejo (RS1); (ii) pouca ou nenhuma ação coletiva (GS6); (iii) a pesca de pequena escala é previsível (RS7); (iv) a elevada mobilidade dos recursos pesqueiros prejudica a autoorganização (RU1); (v) o número de usuários não é elevado e impacta de forma limitada o sistema de recurso (U1); (vi) a falta de liderança e regras locais afetam negativamente o estabelecimento de sistemas de gestão (U5 e U6); (vii) usuários não compartilham conhecimento comum sobre o sistema de recurso (U7) e tomam decisões que podem reduzir a produtividade do sistema (RS5); e (viii) os usuários são pouco dependentes do sistema de recursos para sua subsistência (U8). Estas suposições levam a uma previsão empírica de sobrepesca e a degradação do sistema de recurso.

Para o cenário de sustentabilidade socioecológica, maximizamos as premissas estabelecidas no cenário de insustentabilidade socioecológica visando um sistema autoorganizado: (i) o sistema de recursos envolve áreas de manejo que permitem um zoneamento ou descentralização da pesca (RS1); (ii) existem regras coletivas de gestão dos recursos (GS6); (iii) a pesca de pequena escala é previsível (RS7); (iv) usuários contri- 

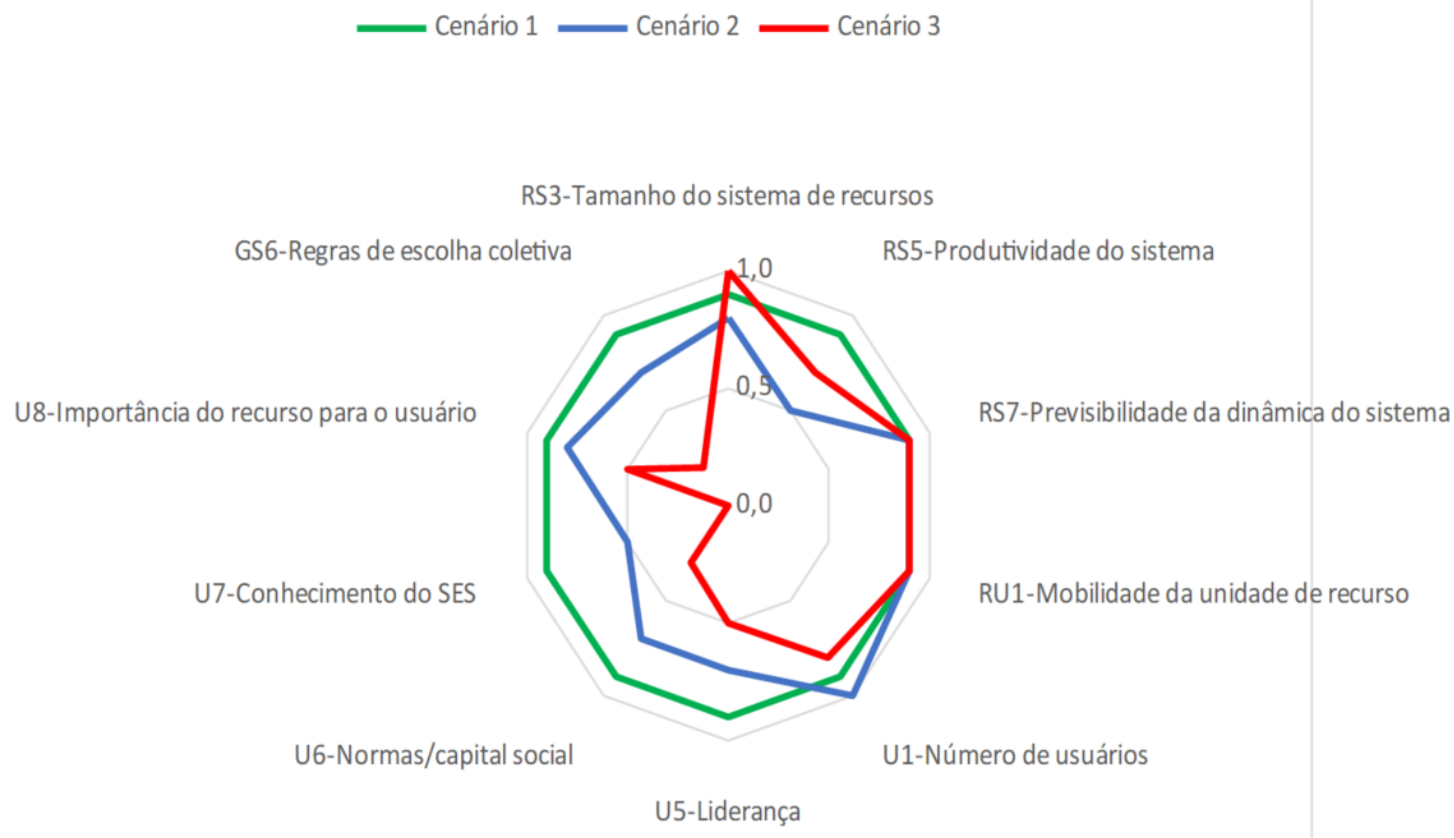

Figura 3. Visualização multidimensional dos cenários de acordo com as notas atribuídas pelos subsistemas. Cenários: (1) Sustentabilidade socioecológica, (2) Insustentabilidade social e cultural, e (3) Insustentabilidade socioecológica.

buem de forma significativa para o sistema de gestão (U1); (vi) a presença de liderança e regras locais impacta positivamente no estabelecimento de medidas de manejo (U5 e U6); (vii) usuários compartilham conhecimento comum sobre o sistema de manejo (U7) e tomam decisões que melhoram a produtividade do sistema (RS5); e (viii) os usuários são dependentes do sistema de recursos para sua subsistência (U8).

O cenário de insustentabilidade social e cultural é baseado na premissa de que os recursos em questão, mesmo que abundantes e com alta produtividade, não fazem parte do uso histórico das populações tradicionais e cuja organização social não possui as bases para o manejo daquele recurso especificamente. Um exemplo típico é o caso do pirarucu (Arapaima gigas), recurso pesqueiro tradicional no estados do Amazonas e Pará e sobreexplorado no final dos anos 1990, porém, com estoques pristinos na região do Alto Purus, estado do Acre. Todavia, as bases de organização social na região do Alto Purus apresentaram dificuldades de lidar com este recurso que não fazia parte de seu histórico de uso. As comunidades ribeirinhas na região estão mais relacionadas com as cadeias produtivas ligadas com a agricultura e recursos florestais, o que se reflete nas 
políticas públicas e apoio institucional existentes na região.

Os cenários nos permitem avaliar o desempenho de sistemas socioecológicos de manejo e podem orientar um roteiro para o desenvolvimento sustentável. Por exemplo, o estabelecimento dos acordos coletivos de pesca (GS6) favorece o capital social (U5) e o conjunto de conhecimentos sobre o sistema socioecológico (U6). Os pescadores tomam decisões coletivas para maximizar a produção sustentável (RS5). Este direito de gestão para o uso continuado de um recurso pode estimular os usuários locais a buscar objetivos de longo prazo (SCHLAGER e OSTROM, 1992).

\section{Conclusões}

Conforme proposto neste artigo, uma abordagem metodológica de aplicação rápida, semiquantitativa, para a identificação dos limiares para a cooperação sustentável, de acordo com as variáveis-chave elencadas por Ostrom (2009) pode ser útil na identificação das condições mínimas a serem observadas na dinâmica dos recursos naturais, da organização social, da economia e das instituições para que os mecanismos de cooperação de um determinado SES sejam sustentáveis.

Logo, do ponto de vista da gestão dos recursos pesqueiros, ao promover a gestão participativa da pesca de pequena escala na Amazônia, é possível tomar as variáveis do SES como referência para verificar se existem as condições mínimas para que a cooperação seja possível. Ou, por outro lado, promover ações que criem estas condições. Neste sentido, tais ações devem ser planejadas considerando que um determinado SES está inserido em um contexto multiescalar e dinâmico, onde conceitos como o de panarquia (GUNDERSON \& HOLLING, 2002; ALLEN et al., 2014) e governança ambiental transformativa (CHAFFIN et al., 2016) devem ser levados em consideração para que tais sistemas possam ser tanto resilientes a mudanças indesejáveis como também capazes de absorver as inovações que os tornem mais eficientes.

Todavia, esta proposta metodológica deve ser aplicada apenas em um contexto onde estudos interdisciplinares que possam avaliar o referido SES de forma ampla ainda não tenham sido realizados. Neste sentido, a avaliação de variáveis-chave proposta neste artigo pode auxiliar na elaboração de hipóteses e perguntas para tais estudos. 


\section{Referências}

AGRAWAL, A. 2001. Common property institutions and sustainable governance of resources. World Development 29(10): 1649-1672.

AGRAWAL, A., e A. CHHATRE. 2006. Explaining success in the commons: community forest governance in the Indian Himalaya. World Development 34(1): 149-166.

ALLEN, C. R., D. G. ANGELER, A. S. GARMESTANI, L. H. GUNDERSON e C. S. HOLLING. 2014. Panarchy: theory and application. Ecosystem 17:578-89

ANDERIES, J. M., e M. A. JANSSEN. 2013. Sustaining the commons. Center for the Study of Institutional Diversity. Arizona State University, Arizona. 167p.

ASAH, S. T. 2008. Empirical Social-Ecological System analysis: From theoretical framework to latent variable structural equation model. Environmental Management 42: 1077-1090.

ASWANI, S., G. G. GURNEY, S. MULVILLE, J. MATERA, e M. GURVEN. 2013. Insights from Experimental Economics on Local Cooperation in a Small-Scale Fishery Management System. Global Environmental Change 23 (6): 1402-1409.

BASURTO, X., e E. OSTROM. 2009. Beyond the Tragedy of the Commons. Economia delle fonti di energia e dell ambiente 52 (1): 35-60.

BAULER, T. 2012. An analytical framework to discuss the usability of (environmental indicators for policy. Ecological Indicators 17: 38-45.

BERKES, F., J. COLDING, e C. FOLKE. 2000. Rediscovery of traditional ecological knowledge as adaptive management. Ecological Applications 10: 1251-1262.

BERKES, F. 2009. Evolution of co-management: role of knowledge generation, bridging organizations and social learning. Journal of Environmental Management 90: 1692-1702.

BEROYA-EITNER, M. A. 2016. Ecological vulnerability indicators. Ecological Indicators 60: 329-334.

BOILLAT, S., F. M. SCARPA, J. P. ROBSON, I. GASPARRI, T. MITCHELL AIDE; A. P. D. AGUIAR, L. O. ANDERSON, M. BATISTELLA, M. G. FONSECA, C. FUTEMMA, H. R. GRAU, S. MATHEZ-STIEFEL, J. P. METZGER, J. P. H. B. OMETTO, M. A. PEDLOWSKI, S. G. PERZ, V. ROBIGLIO, L. SOLER, I. VIEIRA, e E. S. BRONDÍZIO. 2017. Land System Science in Latin America: challenges and perspectives. Current Opinions in Environmental Sustainability 26-27: 37-46.

BRONDÍZIO, E. S., E. OSTROM, e O. YOUNG. 2009. Connectivity and the Governance of Multilevel Socio-ecological Systems: The Role of Social Capital. Annual Review of Environment and Resources 34: 253-78.

BUTLER, J. R. A, A. TAWAKE, T. SKEWES, L. TAWAKE, e V. MCGRATH. 2012. Integrating traditional ecological knowledge and fisheries management in the Torres Strait, Australia: the catalytic role of turtles and dugong as cultural keystone species. Ecology and Society 17(4): 34.

CARPENTER, S. R., B. WALKER, M. J. ANDERIES, e N. ABEL. 2001. From metaphor to measurement: Resilience of what to what? Ecosystems 4: 765-781. 
CASTELLO, L., J. P. VIANA, e M. PINEDO-VASQUEZ. 2011. Participatory conservation and local knowledge in the Amazon várzea: the pirarucu management scheme in Mamirauá. In: PINEDO-VASQUEZ, M., M. L. RUFFINO, C. PADOCH, E E. S. BRONDÍZIO (Eds). The Amazon Varzea: the decade past and the decade ahead. New York: Springer-Verlag. pp. 261-276.

CINNER, J. E., T. R. MCCLANAHAN, M. A. MACNEIL, N. A. J. GRAHAM, T. M. DAW, A. MUKMININ, D. A. FEARY, A. L. RABEARISOA, A. WAMUKOTA, N. JIDDAWI, S. J. CAMPBELL, A. H. BAIRD, F. A. JANUCHOWSKI-HARTLEY, S. HAMED, R. LAHARI, T. MOROVE, e J. KUANGE, J. 2012. Co-management of Coral Reef Social-Ecological Systems. Proceedings of the National Academy of Sciences of the United States of America 109(14): 5219-5222.

CHAFFIN, B. C., A. S. GARMESTANI, L. H. GUNDERSON, M. H. BENSON, D. G. ANGELER, C. A. ARNOLD, B. COSENS, R. K.CRAIG, J. B. RUHL e C.R. ALLEN. 2016. Transformative environmental governance. Annual Review of Environment and Resources, 41:1, 399-423

CONNOR R, e S. DOVERS. 2004. Instituional change for sustainable development. Edward Elgar, Cheltenham. 264p.

COX, M., G. ARNOLD, e S. V. TOMÁS. 2010. A review of design principles for communitybased natural resource management. Ecology and Society 15(4): 38. CRAMPTON, W. G. R., L. CASTELLO, e J. P. VIANA. 2004. Fisheries in the Amazon várzea: Historical trends, current status, and factors affecting sustainability. In SILVIUS, K., R. BODMER, e J. M. V. FRAGOSO (Eds.). People in nature: Wildlife conservation in South and Central America New York: Columbia University Press, 16-95.

DELANEY, A. E., e M. Hadjimichael. 2017. Forming perceptions and the limits to public participation on ocean commons: evidence from a citizens jury workshop. International Journal of the Commons 11(1): 200-219.

DEY, I. 1993. Qualitative data analysis: a user-friendly guide for social scientists. Routledge, New York. 304p.

DURAIAPPAH, A.K., S. T. ASAH, E. S. BRONDÍZIO, N. KOSOY, P. O’FARREL, A-H PRIEUR-RICHARD, e K. TAKEUCHI. 2014. The New Commons: Matching the MisMatches. Current Opinion in Environmental Sustainability 7: 94-100.

ERNST, B, J. CHAMORRO, P. MANRÍQUEZ, J. M. L. ORENSANZ, A. M. PARMA, J. POROBIC, e C. ROMÁN. 2013. Sustainability of the Juan Fernández Lobster Fishery (Chile) and the Perils of Generic Science-Based Prescriptions. Global Environmental Change 23(6): 1381-1392.

FOLKE, C., T. HAHN, P. OLSSON, e J. NORBERG. 2005. Adaptive governance of socialecological systems. Annual Review of Environment and Resources 30:441-473.

FREY, U. J., e H. Rusch. 2013. Using artificial neural networks for the analysis of socialecological systems. Ecology and Society 18(2):40.

GARIBALDI, A., e N. TURNER. 2004. Cultural keystone species: implications for ecological conservation and restoration. Ecology and Society 9(3):1. 
GARRETTA, A., P. MACMULLEN, e D. Symes. 2012. Fisheries as learning systems: Interactive learning as the basis for improved decision making. Fisheries Research 127128: $182-187$.

GUNDERSON, L. H. e C. S. HOLLING (Eds.). 2002. Panarchy: understanding transformations in human and natural systems. Washington,DC: Island Press.

GUTIÉRREZ, N. L., R. HILBORN, e O. DEFEO. 2011. Leadership, social capital and incentives promote successful fisheries. Nature 470: 386.

HARDIN, G. 1968. The tragedy of the commons. Science 162: 1243-1248.

HINKEL, J, P. W. G. BOTS, e M. SCHLÜTER. 2014. Enhancing the Ostrom SocialEcological System Framework through Formalization. Ecology and Society 19(3): 51.

HINKEL, J, M. E. COX, M. SCHLÜTER, C. R. BINDER, e T. FALK. 2015. A Diagnostic Procedure for Applying the Social-Ecological Systems Framework in Diverse Cases. Ecology and Society 20(1): 32.

IBAMA (2003) Instrução Normativa no 29. IBAMA, Brasília.

INGOLD, T. 2011. Being alive: Essays on movement, knowledge and descrioption. London: Routledge. 288p.

JANSSEN, M.A. 2002. Complexity and Ecosystem Management: The Theory and Practice of Multi-agent Systems. Edward Elgar, Massachusetts. 360p.

JOHANNES, R. E. 1998. Government-supported, village-based management of marine resources in Vanuatu. Ocean \&Coastal Management 40:165-186.

JOHANNES, R. E. 2002. The renaissance of community-based marine resource management in Oceania. Annual Review of Ecology and Systematics 33:317-340.

JOHANNES, R. E., M. M. R. FREEMAN, e R. J. HAMILTON. 2000. Ignore fishers' knowledge and miss the boat. Fish and Fisheries 1:257-271.

KIRK-LAWLOR, N., e S. ALLRED. 2017. Group Development and Integration in a CrossDisciplinary and Intercultural Research Team. Environmental Management 59 (4): 665683.

KITTINGER, J. N., E. M. FINKBEINER, N. C. BAN, K. BROAD, M. H. CARR, J. E. CINNER, S. GELCICH, M. L. CORNWELL, J. Z. KOEHN, X. BASURTO, R. FUJITA, M. R. CALDWELL, e L. B. CROWDER. 2013. Emerging frontiers in social-ecological systems research for sustainability of small-scale fisheries. Current Opinion in Environmental Sustainability $5:$ 1-6.

KHOLER, F., e E. S. BRONDÍZIO. 2017. Considering the needs of indigenous and local populations in conservation programs. Conservation Biology 31(2): 245-251.

LEVIN, S.A. 1992. The problem of pattern and scale in ecology. Ecology 73 (6):19431967.

LONDON, S., M. L. ROJAS, M. M. I. MARTIN, F. SCORDO, M. A. H. CISNEROS, M. L. BUSTOS, G. M. E. PERILLO, e M. C. PICCOLO. 2017. Characterization of an artisanal fishery in Argentina using the social-ecological systems framework. International Journal of the Commons 11(1): 1-69. 
MARSHALL, N. A. 2007. Can policy perception influence social resilience to policy change? Fisheries Research 86: 216-227.

MCGINNIS, M., e E. OSTROM. 2014. Social-Ecological System Framework: Initial Changes and Continuing Challenges. Ecology and Society 19(2): 30.

MACNEIL, M. A., e J. E CINNER. 2013. Hierarchical Livelihood outcomes among CoManaged Fisheries. Global Environmental Change 23(6): 1393-1401.

NUNAN, F., J. LUOMBA, C. LWENYA, E. YONGO, K. ODONGKARA, e B. NTAMBI. 2012. Finding space for participation: fisherfolk mobility and co-management of Lake Victoria fisheries. Environmental Management 50: 204-216. OLSSON, P., C. FOLKE, e F. BERKES. 2004. Adaptive co-management for building resilience in social-ecological system. Environmental Management 34(1): 75-90.

OSTROM, E. 1990. Governing the commons - the evolution of institutions for collective action. Cambridge University Press, Cambridge. 280p.

OSTROM, E. 2007. A diagnostic approach for going beyond panaceas. Proceedings of the National Academy of Sciences 104(39): 15181-15187.

OSTROM, E., M. A. JANSSEN, e J. M. ANDERIES. 2007. Going beyond panaceas. Proceedings of the National Academy of Sciences 104(39): 15176-15178.

OSTROM, E. 2009. A general framework for analyzing sustainability of social-ecological systems. Science 325: 419-422.

OSTROM, E. 2011. Background on the Institutional Analysis and Development Framework. Policy Studies Journal 39(1): 7-27.

OTEROS-ROZAS, E., R. ONTILLERA-SÁNCHEZ, P. SANOSA, E. GÓMEZBAGGETHUN, V. REYES-GARCÍA, e J. A. 2013. Traditional ecological knowledge among transhumant pastoralists in Mediterranean Spain. Ecology and Society 18(3):33.

PETURSDOTTIR, T., O. ARNALDS, O., S. BAKER, L. MONTANARELLA, e A. L. ARADÓTTIR. 2013. A social-ecological system approach to analyze stakeholders interactions within a large-scale rangeland restoration Program. Ecology and Society 18(2): 29.

POMEROY, R. S., B. M. KATON, e I. HARKES. 2001. Conditions affecting the success of fisheries co-management: lessons from Asia. Marine Policy 25: 197-208.

PRITCHETT, L., e M. WOOLCOCK. 2004. Solutions when the solution is the problem: Arraying the disarray in development. World Development 32: 191-212. RAYMOND, C. M., I. FAZEY, M. S. REED, L. C. STRINGER, G. M. ROBINSON, e A. C. EVELY. 2010. Integrating local and scientific knowledge for environmental management. Journal of Environmental Management 91(8): 1766-1777.

SINGH, R. K., H. R. MURTY, S. K. GUPTA, e A. K. 2012. An overview of sustainability assessment methodologies. Ecological Indicators 15:281-299.

SCHLAGER, E., e E. OSTROM. 1992. Property rights regimes and natural resources: a conceptual analysis. Land Economics, 68(3): 249-262.

STERNLIEB F., R. P. BIXLER, H. HUBER-STEARNS, C. HUAYHUACA. 2013. A question of fit: Reflections on boundaries, organizations and social-ecological systems. Journal of 
Environmental Management 130:117-125.

STEPHENSON, R. L., S. PAUL, M. WIBER, E. ANGEL, A. J. BENSON, A. CHARLES, O. CHOUINARD, M. CLEMENS, D. EDWARDS, P. FOLEY, L. JENNINGS, O. JONES, D. LANE, J. MCISAAC, C. MUSSELLS, B. NEIS, B. NORDSTROM, C. PARLEE, E. PINKERTON, M. SAUNDERS, K. SQUIRES, e U. R. SUMAILA. 2018. Evaluating and implementing social-ecological systems: A comprehensive approach to sustainable fisheries. Fish and Fisheries 1-21.

TERER, T., A. M. MUASYA, F. DAHDOUH-GUEBAS, G. G. NDIRITU, e L. TRIEST. 2012. Integrating local ecological knowledge and management practices of an isolated semi-arid papyrus swamp (Loboi, Kenya) into a wider conservation framework. Journal of Environmental Management 93(1): 71-84.

WALKER, B., S. R. CARPENTER, M. J. ANDERIES, N. ABEL, G. S. CUMMING, M. A. JANSEN, L. LEBEL, J. NORBERG, G. D. PETERSON, e R. PRITCHARD. 2002. Resilience management in social-ecological systems: A working hypothesis for participatory approach. Conservation Ecology 6(1): 14.

WILSON, J., A. HAYDEN, e M. KERSULA. 2013. The governance of diverse, multi-scale fisheries in which there is a lot to learn. Fisheries Research 141: 24-30. 